\title{
Persistencia de las Representaciones Sociales del Cáncer de Mama
}

\section{Persistence of social representation regarding breast cancer}

\author{
Clara V. Giraldo-Mora \\ Facultad de Enfermería, Universidad de Antioquia. Medellín, Colombia. clarag@tone.udea.edu.co \\ Recibido 9 Mayo 2008/Enviado para Modificación 16 Noviembre 2008/Aceptado 15 Julio 2009
}

RESUMEN

Objetivo Comprender las representaciones sociales del cáncer de mama para un grupo de mujeres de la ciudad de Medellín, la influencia en su prevención y en las prácticas de autocuidado.

Metodos Estudio cualitativo con 19 entrevistas semiestructuradas con mujeres adultas que no han tenido cáncer de mama, utilizando el criterio de máxima variación como técnica de muestreo. El análisis se orientó por la teoría fundamentada.

Resultados Algunas mujeres tienen representaciones fisiológicas del cáncer, otras lo hacen desde sus efectos sociales y psicológicos e identifican sus causas con problemas personales y del afecto o hábitos cotidianos como alimentos inadecuados - "una venganza del cuerpo por los abusos que se hacen sobre el'.

Discusión La palabra "cáncer de mama" se asocia a muerte inevitable, terror, sufrimiento, devastación, incurabilidad, impotencia y dolor. Este cáncer tiene fuertes representaciones sociales debido a las severas implicaciones en el aspecto femenino, atractivo y autoimagen.

Conclusiones La persistencia de las imágenes negativas del cáncer, se asocia al mito de los estilos de vida, que responsabiliza al paciente. Nuestro reduccionismo biológico oculta factores ambientales, sociales y políticos. Estamos obsesionados con los peligros y su control y añadimos a estos mensajes, imágenes poderosas como aquella que "una en nueve mujeres desarrollarán el cáncer de mama”, para fomentar la propia responsabilidad; de esa forma, magnificamos los fantasmas del cáncer que nuestras sociedades en desarrollo están tratando de superar y en las que todavía estamos atrapados.

Palabras Clave: Investigación en Enfermería, investigación cualitativa, cáncer de mama, salud de las mujeres, prevención, autocuidado (fuente: DeCS, BIREME).

\section{ABSTRACT}

Objective Understanding the social representation of breast cancer and how it has influenced breast cancer prevention and self-care practice in a group of women from the city of Medellin.

Methods This was a qualitative study using 19 semi-structured interviews with adult females who had not had breast cancer, using maximum variation criterion as sampling technique. The analysis was orientated by grounded theory. 
Results Some women physiologically represented breast cancer while others represented it by its social and psychological effects. They identified its causes with personal and emotional problems and certain daily habits such as inadequate food ("a bodily payback for the abuses which we subject ourselves to").

Discussion The word "breast cancer" was associated with inevitable death, terror, suffering, incurability, devastation, powerlessness and pain. This cancer has strong social representation due to its severe implications for females, their attractiveness and self-image.

Conclusions The persistence of breast cancer's negative image is associated with 'the life-style myth" (1) for which people tend to blame the patient. Our biological reductionism hides environmental, social and political factors. We are obsessed by the dangers and their control (2) and powerful images are added to these messages such as those in which "one out of nine women will develop breast cancer" to foster self-responsibility (2). However, the ghost of cancer in developing societies in which many people are still trapped is magnified and has also yet to be overcome.

Key Words: Nursing research, qualitative research, breast cancer, women's health, prevention, self-care (source: $M e S H, N L M$ ).

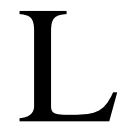

a motivación para esta investigación surgió de la necesidad de una lectura no biomédica de los estudios sobre cáncer en nuestro medio, basada en el reconocimiento de la persistencia de imágenes negativas asociadas al cáncer y al mito de la necesidad de control sobre los factores de riesgo, de diversa índole, que se le asocian $(1,2)$.

En el mundo, anualmente hay más de 10 millones de casos nuevos y más de 6 millones de defunciones por cáncer, es el $12 \%$ del total de muertes. En 1980 estas cifras eran de 6 y 4 millones, respectivamente (3). Los casos aumentarán de 10 millones en el 2000 a 15 millones para el 2020. Las mujeres de países pobres tienen menor supervivencia, debido al alto porcentaje de casos diagnosticados tardíamente (4).

Estas cifras hacen del cáncer de mama, un problema de salud pública; es el tumor maligno más frecuente entre las mujeres (5) (afecta una de cada diez mujeres y representa el $21 \%$ de los nuevos casos (6) en esta población. Las mujeres que padecen la enfermedad afrontan situaciones importantes y difíciles, como: Detección de algún problema en la(s) mama(s), confirmación del diagnóstico, período que antecede al tratamiento, la cirugía, el tratamiento terapéutica subsiguiente con radioterapia, quimioterapia y otros procedimientos (7).

El cáncer es prioritario en el perfil epidemiológico de Colombia (8). Para el año 2002 el cáncer ginecológico representó el 19,6 \% de los casos y el 31,8 \% en el sexo femenino (8). El cáncer de mama fue la segunda neoplasia maligna más 
común en mujeres $(19,7 \%)(10)$. Para ese año se registraron 608 nuevos casos. El promedio de edad fue de 53,9 años y el $40,5 \%$ era menor de 50 años, teniendo mayor ocurrencia en mujeres mayores de 35 años. El $70 \%$ de los casos ingresaron en estadios tardíos (III y IV) y el 5,1 \% en estadios tempranos (in situ y estadio I) (9).

Las representaciones sociales trascienden la esfera de las opiniones, las imagines y las actitudes, son sistemas cognoscitivos con una lógica y lenguaje particulares que descubren y ordenan la realidad (10). Estas son producciones culturales y sociales que afectan nuestras prácticas, conocimiento y relaciones subjetivas (10).

Las representaciones sociales de una enfermedad son determinantes de los comportamientos culturales con respecto a la misma, en las cuales confluyen un complejo conjunto de conocimientos científicos y populares, la escala de valores y otros elementos culturales, como las creencias, que determinan la reacción general de la sociedad y la particular de cada individuo frente a una enfermedad (11).

Las creencias de las personas sobre el cáncer son muy variadas; el cáncer es asociado con muerte, dolor y fetidez (12). Sabemos que la representación social del cáncer es organizada alrededor del sentido subjetivo de muerte, mutilación, vacíos, incapacidad, el fin de la vida social, entre otros. La muerte existe en nuestra sociedad como un sentido, pero como un sentido distante, algo que tiene poco que hacer con nosotros (12). El cáncer hace la muerte presente, inmediata y real.

Existen importantes estudios en el mundo sobre las representaciones sociales del cáncer de mama (13-15), pero en Colombia los hay sobre otros tipos de cáncer $(1,16)$ que fueron puntos de referencia de nuestro estudio, sin embargo que el desarrollo científico de un país debe ser impulsado desde varios nichos investigativos como el desarrollado por la Universidad de Antioquia.

\section{METODOLOGÍA}

El estudio se realizó en el Departamento de Antioquia, entre el 2006 y el 2008, con una perspectiva metodológica cualitativa y con 19 mujeres, entre 18 y 69 años, con diversas características étnicas, educativas, ocupacionales y socio-económicas, a quienes no se les había diagnosticado cáncer de mama, con capacidad para hablar, que en forma voluntaria estuvieron dispuestas a compartir sus pensamientos y a quienes se les garantizó el manejo ético de la información. La técnica de recolección de la información, fue la entrevista semiestructurada (17), concepto que indica encuentros cara a cara entre las entrevistadas y las investigadoras. Para el análisis se siguieron los lineamientos de la teoría fundada (17). La recolección, el análisis y 
la postura teórica se hizo conjuntamente y mantuvieron una relación reciproca durante todo el proceso investigativo.

Para conformar el grupo de informantes, se utilizó la técnica de máxima variación, la cual sugiere incluir mujeres con la mayor diversidad cultural, étnica, social y económica a fin de responder a la heterogeneidad de la población (17). Para la determinación del número de personas a entrevistar, se utilizó el muestreo teórico (18), es decir, que el número de personas se establece partiendo de los conceptos identificados como relevantes para la comprensión del fenómeno (18). Dos entrevistas fueron parte del estudio exploratorio en el cual se comprobó que las preguntas y el acercamiento a las mujeres eran adecuados. La entrevista fue modificada cuando se obtuvo información que permitió ampliar y enfocar el objeto de estudio. El análisis de la investigación se realizó con el Software Atlas-ti. La validez del análisis se rige por los criterios de credibilidad y veracidad (18). Esta validez se logra cuando las categorías emergen de manera natural, se saturan y son validadas por personas que participan como informantes en el estudio (19). Este estudio fue presentado a 6 de las participantes quienes se identificaron con los hallazgos y pidieron mayor difusión entre otras mujeres.

\section{RESULTADOS}

Se le considera una enfermedad catastrófica que acarrea pérdidas importantes, con efecto emocional destructor como el miedo, la desestabilización del proyecto de vida. Creen que existe angustia por deficiente calidad de la atención en salud y sufrimiento para la familia. Así mismo, la ignorancia colectiva e histórica sobre la enfermedad la hace más temible y difícil de llevar.

De igual forma, las mujeres tienen representaciones sobre la prevención y el diagnóstico. En general, reconocen estar desinformadas respecto a la fisiopatología del cáncer; las mujeres lo conciben como "calculitos, piedritas", células que se reproducen anormalmente y van conformando una "bolita", que por su crecimiento va haciendo metástasis y dañando otros órganos; así lo entiende Lina (R 456) (En lo sucesivo se utilizará la inicial " $R$ " como identificación del reglón en que un código empieza dentro de cada entrevista de acuerdo al programa Atlas ti): "hay unas células aquí que forman una bolita, unas células se están produciendo más de la cuenta y entonces, ellas no encuentran más que hacer que seguirse reproduciendo, necesariamente, van a empujar otros tejidos...'(Beatriz R 277, 283). 
El cáncer de mama es considerado una enfermedad catastrófica porque destruye la vida de muchas mujeres, involucra pérdidas importantes y acarrea sufrimiento, por lo que requiere de la elaboración de duelos. Por un lado, se pierde la integridad del cuerpo por el tratamiento (mastectomía, quimioterapia, etc.), por el otro, hay cambios importantes en las funciones y estructuras corporales. Laura (R 184) lo expresa así: "Yo creo que como dije anteriormente, es algo duro y difícil, en el sentido de perder algo y que ese algo involucre la vida, de perder una parte del cuerpo, igual que si te cercenas un dedo a vos igual te va a doler, no lo vas a tener $y$ te va hacer falta, porque siempre va a cumplir una función, entonces es como esa pérdida más que todo". Además, se teme al abandono de la pareja, involucrando el mundo afectivo y sexual y deteriorando su autoestima, porque este cáncer compromete órganos que son, especialmente, erógenos y atractivos para ellas.

Se encontró que no hay preocupación por la perdida de la intimidad si no se ha iniciado la vida sexual. "Cercenar" la mama, significa el principio de la extinción de la sexualidad y de la vida en pareja, algo que puede implicar una forma de ir muriendo. Contrario a lo que sucedería por parte de los hombres, el amor de una mujer por su pareja masculina no se ve afectado negativamente, si éste llegara a tener cáncer; así lo refiere Liliana (R 239): "Es que yo no lo cambiaría, pues si el caso fuera de él yo no lo cambiaría, porque igual lo que uno siente es el amor, pues como la persona que es ya, pero ya un hombre no sé qué pensaría, ya habría que preguntarle a él, pero no sé”.

Adicionalmente, se desestabiliza el proyecto de vida, lo que da lugar a un giro definitivo de las relaciones y del estilo de vida; cambios drásticos en el mundo laboral y social, como expresa Bibiana (R 227): "ya no seguiría el modelaje, pues igual las relaciones ya no serían de amistades y todo, no llevaría la vida que llevo en este momento que es que en fin de semana a rumbear que con los amigos, pues no, no”.

Involucra también, efecto emocional destructor como el miedo a no saber afrontar la enfermedad y sus consecuencias como la metástasis, la deformación, el dolor, la quimioterapia y la muerte. Asimismo, el aislamiento, deseo de esconderse de los otros cuando sufra los efectos secundarios del tratamiento; Liliana (R 227) dice: "Me sentiría como incómoda al ver que se me está como cayendo el pelo, o, pues todo el proceso que trae como la quimioterapia, como te decía ahorita; entonces, no me gustaría que me vieran así".

Existe entre ellas la inquietud sobre si la angustia y el sufrimiento intenso pueden desencadenar ésta dolencia (Doris R 86), piensan que el estrés (Julia R 137), los problemas personales y del afecto tienen relación (Morelia $\mathrm{R}$ 103), dando lugar a la disminución de las defensas del organismo (Lina R 406 y 472) y favoreciendo el desarrollo del cáncer, al igual que algunos hábitos personales 
como uso de brassier y baño con agua caliente (Morelia R 103). Afirman desconocer la relación entre el estilo de vida y las causas de la enfermedad, pero creen que los estilos de vida y alimentación producen efectos en el cuerpo que representan una venganza del cuerpo mismo, pues no se discriminan aquellos que pueden llegar a ser dañinos para la salud; Luz (R 130), una odontóloga cuya secretaria vivió el cáncer de mama, expresa: "se alimentan con cosas que le dan muy duro al organismo y hay una cosa gravísima y es que el organismo toma venganza de todo lo malo que uno le hace. La misma secretaria esa, era de chorizos, de no sé que, y yo le decía: no comas tal cosa, en cambio, hay mucho alimento que uno sabe que le puede servir". Sin embargo, otras mujeres piensan que tener una vida bien cuidada no exime del cáncer, entonces, se preguntan ¿cómo prevenirlo? (Lina R 338). Asimismo, identifican su origen en traumas físicos mal cuidados; (Lina R 490); incertidumbre acerca de la influencia de cuerpos extraños como los "implantes" mamarios (Julia R 137). Algunas consideran que hay mayores riesgos en aquellas mujeres que no han lactado y otras por el contrario, asocian la lactancia con la mastitis y ésta con el cáncer (Lina R 487). Otras estiman que, incluso, puede ser producto de efectos iatrogénicos de medicamentos o como resultado de un factor genético (Lina R 406 y 472). Para algunas mujeres la generación de la enfermedad depende de la voluntad de Dios (Martha R 174), otras por el contrario, consideran que la aparición del cáncer no está relacionada con la religión (Bibiana R 208), ni es un castigo (Morelia R 112).

“Sobre la enfermedad no se habla con nadie..."(Hellen R 202), hay poca información, “...Yo creo que casi nada... Más que todo de pronto uno lee uno que otro documentito por ahí” (Lina R 4), lo que lleva al desconocimiento y temor, pero la experiencia familiar cercanas de la enfermedad estimula el deseo de saber acerca del origen y la manera de detectarla (Morelia R 133). Tienen internalizados estereotipos frente a la percepción del riesgo de cáncer de mama, como lo afirma Morelia (R 30): “...nunca nos va a pasar a nosotras..., que el cáncer le da a una persona de edad o a una persona que nunca se cuida..., es como no tener temor a padecer de la enfermedad".

Frente al diagnóstico, expresan temor a ser juzgadas por no prevenir y, a su vez, a recibir diagnóstico confirmatorio de la enfermedad (Morelia R 120). Adicionalmente, imaginan los procedimientos como dolorosos. En síntesis las representaciones del cáncer de mama en las mujeres del estudio, fueron bastante tenebrosas y denotaron enorme temor a la perdida, a la mutilación, al dolor y la muerte. 


\section{DISCUSIÓN}

Las descripciones más antiguas del cáncer

$\mathrm{Al}$ revisar la literatura se hizo evidente que nuestras representaciones del cáncer están arraigadas en la profundidad de la historia y posiblemente no fácil de modificar, porque el cáncer ha afligido a la gente a través de toda la historia (20-25).

El cáncer de mama no solo es una vieja enfermedad que supera la raza, la clase, el tiempo y el espacio, sino que es un horror conocido en cada cultura y en cada edad (21). Entre los antiguos, el único cáncer conocido era el de mama, pues los médicos no podían ver otros tumores porque las autopsias no fueron socialmente aceptadas hasta el siglo diecisiete (21), mientras que el cáncer de mama, era claramente visible, progresaba de un pequeño nódulo a grandes tumores, causando estragos en la mama y el cuerpo (21).

Es fácil entender por que desde la antigüedad el cáncer ha producido tanto horror, pues en ausencia de los tratamientos modernos, un tumor de mama se dividía rápidamente. Todos los tratamientos en la edad media dieron lugar a una lista de agentes farmacéuticos, así como medicinas extrañas, como cataplasmas de orina o hígado de lobo, que incluso envolvían gritos y conjuros a los dioses. El tratamiento favorito del Galeno era la sangría (21). A pesar de los grandes avances de la medicina, el horror por la mastectomía y el asunto de ser bellas y atractivas es central a sus preocupaciones $(23,24)$. Históricamente hay un profundo arraigo en las creencias religiosas, interpretando el cáncer como castigo, prueba o voluntad divina (21).

En los siglos dieciocho y diecinueve las implicaciones psico-sexuales de la amputación de pecho fueron obscurecidas por el silencio característico del período donde la enfermedad y su cura representan una especie de vergüenza sexual (26). En ese momento, el cáncer de mama salió de la intimidad radical del cuerpo y se medicalizó la sexualidad (26).

El Cáncer ayer, hoy y siempre

El cáncer es una enfermedad terrible, caprichosa e insidiosa (20). Tenemos una percepción distorsionada de ésta porque su mera evocación engendra una penetrante y atemorizante noción de lo que significa la enfermedad (20). "Es una pena que no podamos des-inventar esta palabra" (20). El terror se evidencia en los relatos de las entrevistadas, esta ancestralmente impregnado en nuestro imaginario colectivo (20). Sogtan describe en su libro "La Enfermedad como 
Metáfora" que el cáncer llego a ser el templo de sus propios mitos, lo describe como obsceno, demoníaco y como un predador invencible y macabro que daña todo (25).

Su compleja etiología nos ha llevado a explicaciones que no borran las percepciones erróneas de las causas. Nuestras mentes, por esa dura realidad, necesitan aliviar el sentimiento de culpa, inculpando a los malos hábitos, a los malos genes, la comida que comemos, el aire que respiramos. La dolorosa realidad es que no hay un santo grial, una cura mágica o reparación inmediata (20).

Las metáforas del cáncer están profundamente ancladas en los seres humanos $(20,21,25)$, mas aun, el cáncer favorece la creación de metáforas y es objetiva y prácticamente imposible liberarnos y desmitificar las metáforas del cáncer, razón por la cual, se titulo este estudio persistencia en la representación (1).

Se le asocia con temibles consecuencias cercanas a la muerte como la mutilación o amputación también ha sido asociado con dolores insoportables (20), además ha sido considerado como una muerte no estética (20). Cuando el cáncer aflige a alguien, la persona se preguntaban ¿por qué yo? (20). Nada de esto es ajeno a las mujeres de nuestro estudio a quienes les preocupa perder la vida o una parte del cuerpo, solo que ahora el tratamiento de la medicina moderna les hace temer a la deformación por la mastectomía, a la perdida del cabello y soportar los efectos de la quimioterapia.

\section{Representaciones Sociales del Cáncer en Mujeres Sanas}

Las imágenes en los medios de comunicación condensan el sentido compartido que permite a los miembros de una misma cultura interpretar el impacto poderoso en nuestras actitudes, valores, creencias y comportamientos (27). Se encontró que persisten las imágenes sobre la culpa del paciente y la ausencia de una representación realista de los factores de riesgo como la edad, la raza y la pertenencia étnica, aunque la inculpación, subordinación y desempoderamiento han disminuido (27). La construcción social de la belleza femenina y los estereotipos de género parecen dominar la exactitud en la creación de estas imágenes, concentrándose en el aspecto físico, en lugar de las preocupaciones de la salud (27).

\section{Representaciones Sociales del Cáncer de Mujeres con Cáncer}

En la persona enferma de cáncer, la muerte llega a tener un sentido subjetivo, apareciendo como una realidad emocional que está relacionada con nosotros (28). 
El sentido personal de la muerte es articulado dentro de otros sentidos subjetivos, configurando un sistema poderoso responsable del camino del cual la enfermedad es percibida por la gente. Además aparece una capacidad para apreciar los nuevos aspectos de la vida diaria (28). El impacto subjetivo del cáncer es tan grande, que esto facilita un cambio del estilo de vida que proporciona un nuevo acercamiento a la vida $(12,28,29)$.

Las representaciones sociales del cáncer influyen en la participación de la mujer en su tratamiento y el cuidado de la enferma (28). Se ha encontrado que se representa como una enfermedad incurable que afecta la esfera psicológica, familiar y social de la mujer y la lleva a repensar sus valores, aspectos espirituales, sociales y culturales, incluyendo la apariencia física. Nuestra sociedad, también resalta la relación entre las mamas con la sensualidad y la sexualidad, siendo explotada en un universo erotizado (28). Nuestras entrevistadas hablaron de su preocupación por las interferencias en la intimidad, pudiendo inferir el drama que viven las mujeres enfermas.

El cáncer es una dolencia que preocupa a la sociedad como un todo y una marca social del sujeto (28) Nuestras entrevistadas asociaron el cáncer con muerte, dolor y un recelo a ser estigmatizadas. Algunos autores observaron que el cáncer de mama es una dolencia que se escribe en lo femenino, pues la figura es el universo de la mujer $(28,30)$. El cáncer de mama imprime en las personas una marca socio-corporal, pues el tratamiento es agresivo como las cirugías mutiladoras o la quimioterapia que desfigura a la mujer con la caída del cabello (20).

Un estudio de mujeres con cáncer en Hong Kong usando la hermenéutica filosófica de Gadamer, encontró que ellas creían que la desarmonía podía causar cáncer, además de la tensión, emoción, dieta, ejercicio, genética y destino (29), hallazgo que coincide con nuestros datos.

Al comparar las representaciones del cáncer de mama de pacientes con mujeres sanas, se ha encontrando que las mujeres sanas tienen una representación más desfavorable y exagerada de la enfermedad (30). El cáncer es la condición más dolorosa, menos entendida médicamente y menos evitable que la enfermedad cardiaca, diabetes o el SIDA (30). Entre esas creencias esta la que señala que ingesta de hormonas femeninas puede desarrollar cáncer de mama (31). Las personas interpretación en términos de su sistema de creencia, pero la certeza en esta creencias es ilusoria por que la información o eventos y percepción de las mujeres sobre ingesta hormonal han cambian en los últimos 30 años (31). 
También en nuestras entrevistadas aparecía la preocupación de que el riesgo de cáncer fuera mayor para la mujeres que consumían anticonceptivas o estaban en terapias de remplazo hormonal.

La persistencia de las imágenes negativas del cáncer tiene que ver con "El mito de los estilos de vida" (1), criticado y reconocido por varios autores (2, $20,21)$ y que también hacen parte de nuestros hallazgos, en tanto las personas tienden a culpar a los pacientes. Los nuevos enfoques de riesgo epidemiológico y estadístico son responsables de la representación negativa que tenemos del riesgo del cáncer de mama. Estamos obsesionados con las posibilidades del peligro y su control (2). En la sociedad contemporánea, se cree que los acontecimientos son gobernados según leyes físicas, que pueden ser predichos y potencialmente controlados por los individuos. El uso de la palabra 'riesgo' tiene el efecto retórico que crear un aura de neutralidad y legitimidad científica del discurso social que hace responsables a los individuos (2).

Una de la dificultad principal es hacer medición del concepto de riesgo. Existe una carencia de escala de medición exacta de éste y esto no es simplemente una diferencia entre lengua técnica y lega, sino en la decisión crítica de a quien se asigna la culpa (2). Podemos estar seguros que lo hacen para animar a mujeres a someterse a mamografías anuales y engendrar la auto responsabilidad de la salud (2), pero también se magnifican los fantasmas del cáncer que la sociedad en desarrollo está tratando de superar y en las cuales todavía nos hallamos atrapados

Agradecimientos. Articulo producto de la Investigación Representaciones Sociales del Cáncer de Mama para un Grupo de Mujeres de Medellín y el Área Metropolitana. Colombia, 2008. Realizado por el Grupo de Investigación Salud de las Mujeres, Facultad de Enfermería, Universidad de Antioquia; con financiamiento del Comité para el Desarrollo de la Investigación-CODI- Universidad de Antioquia.

\section{REFERENCIAS}

\footnotetext{
1. Medina AM. Representaciones del Cáncer en Estudiantes de Medicina: Implicaciones para la Práctica y la Enseñanza. Revista de Salud Pública (Bogotá). 2007; 9(3); 369-378.

2. Lock M. Breast cancer: reading the omens. Anthropology Today. 1998; 14(4):7-16.

3. Organización Mundial de la Salud. Programas Nacionales de Control del Cáncer: Políticas y Pautas para la Gestión. [Internet] Organización Mundial de la Salud. Disponible en: http:// www.paho.org/Spanish/AD/DPC/NC/pcc-NCCP.pdf Consultado: 7 agosto 2005.

4. Wiesner $\mathrm{C}$. Determinantes psicológicos, clínicos y sociales del diagnóstico temprano del cáncer de mama. Revista Colombiana de Cancerología. 2007; 11(1): 13-22.
} 
5. Radice D, Redaelli A. Breast cancer management: quality of life and cost considerations. Harmacoeconomics 2003; 21(6): 383-396.

6. Medicina TV. Com. Sigue la lucha contra el cáncer de Mama: La Asociación Española Contra el Cáncer (AECC) celebra cada 19 de Octubre el Día Contra el Cáncer de Mama [Internet] Medicina TV. com Salud. Disponible en http://salud.medicinatv.com/reportajes/ muestra.asp?id=589. Consultado: 5 de agosto 2005.

7. Paiva F, Mesquita E, Magalhães R, Mamede M. $O$ soporte social para atender as necessidades de mulheres mastectomizadas. Rev. Bras. Cancerol 1998; 44 (3): p.231-238.

8. Ministerio de Salud. Colombia. III Estudio Nacional de Salud Bucal y II Estudio Nacional de factores de riesgo de enfermedades crónicas- ENFERC II Serie Documentos Técnicos. Colombia. El ministerio; 1999. p. 31.

9. Pardo C, Murillo R, Piñeros M, Castro MA. Casos nuevos de cáncer en el Instituto Nacional de Cancerología, Colombia, 2002. Revista Colombiana de Cancerología 2003; 7(3): 10.

10. Moscovici S. Social Representations: Essays in Social Psychology. New York: NYU Press. 2001. p. 313.

11. Jodelet D, Guerrero A. Develando la Cultura. $1^{\underline{a}}$ ed. México: Universidad Nacional Autónoma de México; 2000. p.8.

12. González F. A qualitative approach to the study of social representations: the epistemological and ontological basis. VIII International Conference on Social Representations. Social Representation Media and Society. 2006. Disponible en: http://www.europhd.net/8thICSR/ presentations.html Consultado: Enero, 2009.

13. Wilkinson S. Breast cancer: feminism, representations and resistance - a commentary on Dorothy Broom's 'reading breast cancer'. Health. 2001:, 5(2), p. 269-277.

14. Thorne S, Murray C. Social constructions of breast cancer. Health Care for Women International. 2000; 21 p.141-159.

15. LimaA. Social representations of female breast cancer, its etiology and treatment. Pontifícia Universidade Católica do Rio de Janeiro - Puc-Rio. 2005. Disponible en: http://www.maxwell.lambda.ele.pucrio.br/cgi-bin/db2www/PRG_0651.D2W/SHOW?CdLinPrg=pt\&Cont=6059:en Consultado: Enero 16 de 2009.

16. Wiesner C, Vejarano M, Caicedo JC, Murillo SL, Cendales R. La citología de cuello uterino en Soacha, Colombia: representaciones sociales, barreras y motivaciones. Rev. Salud pública. 2006; 8(3):1.

17. Strauss, A, Corbin, J. Bases de la investigación cualitativa: Técnicas y procedimientos para desarrollar la teoría fundamentada. Medellín: Universidad de Antioquia; 2002. p.13-14.

18. Taylor S, Bogdan R. Introducción a los métodos cualitativos de investigación. Barcelona: Paidós Ibérica; 1992. p. 343.

19. Lagoueyte M. La citología vaginal mirada desde las mujeres. Revista Investigacion y Educación en Enfermeria 2002; 20 (2): 60.

20. Greaves M. The Evolutionary Legacy. Oxford University Press; 2002. p. 276.

21. Olson JS. Bathsheba's breast: women, cancer \& history. Baltimore: Johns Hopkins University Press; 2002. p. 455.

22. Leonard RC. Cancer: the evolutionary legacy. Journal of the Royal Society of Medicine. 2001; 94(6):308-309.Disponible en: http://www.pubmedcentral.nih.gov/ articlerender.fcgi?artid=1281537 Consultado: Julio, 2005.

23. Lane S. Cancer history. Rare Cancer Alliance. 2000. Disponible en: http://www.rare-cancer.org/ history-of-cancer.html Consultado: Diciembre, 2007.

24. American Cancer Society. The history of cancer. American Cancer Society. 2002. Disponible en:http: //www.cancer.org/docroot/cri/content/cri_2_6x_the_history_of_cancer_72.asp?sitearea=cri Consultado: Diciembre, 2007.

25. Sontag, S. Illness as metaphor and AIDS and its metaphors. Anchor; 1989. p. 192.

26. Epstein J. Writing the unspeakable: Fanny Burney's mastectomy and the fictive body. Representations. 1986; (16): 131-166. 
27. Andsager J, Stacey J, Powers A. Patient-Blaming and Representation of Risk Factors in Breast Cancer Images. Women \& Health; 2001. 31(2) p 37-70.

28. Lima R, Ferreira M. The social representation of the woman about the cancer: implications for the nursing care. Online Brazilian Journal of Nursing [Internet]. 2003; 2(2). Disponible en: www.uff.br/nepae/objn202limaferreira.htm Consultado: Octubre, 2006.

29. Simpson P. Hong Kong families and breast cancer: beliefs and adaptation strategies. PsychoOncology. 2005; 14: 671-683.

30. Anagnostopoulos F, Spanea E. Assessing illness representations of breast cancer A comparison of patients with healthy and benign controls. Journal of Psychosomatic Research. 2005; 58(4): 327-334.

31. Breakwell G, Barnett J. A hazard sequence. Hazard sequences: social processes amplifying and attenuating health risk scares. G.M Social Psychology European Research Institute 2001 Disponible en: http://www.lps.univ-savoie.fr/dataLPS/colloques/datasPPH/ diaporama/Breakwell.pdf Consultado: Noviembre, 2007. 